\title{
IDENTIFICATION OF THE HEAT TRANSFER COEFFICIENT AT THE CHARGE SURFACE HEATED ON THE CHAMBER FURNACE
}

\begin{abstract}
The inverse method was applied to determine the heat flux reaching the charge surface. The inverse solution was based upon finding the minimum of the error norm between the measured and calculated temperatures. The charge temperature field was calculated with the finite element method by solving the heat transfer equation for a square charge made of $15 \mathrm{HM}$ steel heated on all its surfaces. On the basis of the mean value of heat flux, the value of the heat transfer coefficient at each surface was determined depending on the surface temperature of the material heated.

Keywords: heat transfer coefficient, inverse method, finite element method
\end{abstract}

\section{Introduction}

The modelling of heat transfer in industrial furnaces is one of the more challenging issues is. It is necessary to analyse the system: charge - furnace chamber - environment. The solution to the heat transfer equation does not usually pose problems, as long as the physical properties of the material and the boundary conditions on the surfaces of the materials heated are known. Due to the high temperatures, fuel combustion and the flue gas movement in the furnace, accurate solutions should not only include the heat conduction in the charge, but in the whole furnace chamber taking into account flue gas movement and fuel combustion reactions. Such a comprehensive numerical model of the heating process can be created in leading packages for numerical fluid mechanics. Unfortunately, the computing time arising from the large number of equations necessary to be solved in each iteration is usually very long, and the quality of the obtained results is difficult to be assessed. Consequently, to obtain results in a short time, models are simplified. At the preliminary computing stage only, the combustion process in the burner is considered, resulting in the flue gas composition being set later as an input parameter for furnace calculations (but what is in the furnace?) It allows the analysis of the heating process to be reduced to linked problems of heat transfer and fluid mechanics. Unfortunately, despite this simplification, this method is still ineffective with regards to time. Therefore, it seems reasonable to look for a method which would define boundary conditions without the need to consider all phenomena occurring during charge heating in chamber furnaces fired with natural gas. These possibilities are created by the application of inverse methods to identify the boundary conditions.
Due to the high complexity of effects occurring in heating furnaces chambers, the use of inverse methods was only possible on two planes. The first of these methods belongs to a group called IRP (Inverse Radiation Problem). It allows the temperature distribution, heat flux density or radiative properties of the intermediate medium to be modelled on the basis of temperature measurement in the heating furnace chamber. Many researchers have been developing these methods for a space filled with an intermediate medium in radiation mechanisms [1-4]. Thus obtained parameters may be used as the boundary condition to solve heat conduction equations in the material heated. The other group of methods called IHCP (Inverse Heat Conduction Problem) allows the boundary condition of heat transfer at the material surface to be determined on the basis of temperature measurements at its surface [5-8]. For determining boundary conditions of heat transfer in heating furnaces with indirect methods, it turns out that the correct determination of the heat transfer coefficient involves serious difficulties, despite the relatively well developed methods of inverse analysis $[9,10]$. This arises mainly from the fact that in high-temperature furnaces fired with natural gas, it is difficult to achieve credible values for the surface and atmosphere temperatures. Consequently, the obtained values of the heat transfer coefficient when such high heat fluxes reach the charge surface may be subjected to high fluctuations, caused, among others, by measurement errors. Work on this subject was specifically described in [11]. The heat transfer coefficient was determined on the surface of a cooler through which flue gas with a temperature of $1200 \mathrm{~K}$ was flowing. It was assumed that the properties of the flue gas and the walls were constant over that time. The temperature distribution used in the inverse analysis was simulated by a direct (or analytical) solution, which significantly simplified the solution.

\footnotetext{
AGH UNIVERSITY OF SCIENCE AND TECHNOLOGY, FACULTY OF METALS ENGINEERING AND INDUSTRIAL COMPUTER SCIENCE, AL. MICKIEWICZA 30, 30-059 KRAKOW, POLAND

* Corresponding author: agoldasz@metal.agh.edu.pl
} 


\section{The heat conduction model}

The heat conduction model in the heated charge was based on the solution with the finite element method of the heat conduction equation, which in Cartesian coefficients assumes the form:

$$
\frac{\partial T}{\partial \tau}=\frac{1}{\rho c}\left[\begin{array}{l}
\left.\frac{\partial}{\partial x_{1}} \lambda\left(\frac{\partial T}{\partial x_{1}}\right)+\frac{\partial}{\partial x_{2}} \lambda\left(\frac{\partial T}{\partial x_{2}}\right)+\right] \\
+\frac{\partial}{\partial x_{3}} \lambda\left(\frac{\partial T}{\partial x_{3}}\right)
\end{array}\right]+\frac{\dot{q}_{v}}{\rho c}
$$

where:

$\lambda$ - thermal conductivity, $\mathrm{W} /(\mathrm{m} \cdot \mathrm{K})$;

$x_{1}, x_{2}, x_{3}-$ Cartesian coordinates, $\mathrm{m}$;

$\rho-$ density, $\mathrm{kg} / \mathrm{m}^{3}$;

$c-$ specific heat, $\mathrm{J} /(\mathrm{kg} \cdot \mathrm{K})$;

$\dot{q}_{v}-$ capacity of the external heat source, $\mathrm{W} / \mathrm{m}^{3}$;

$\tau$ - time, $\mathrm{s}$.

The temperature field $T(x, y, z, \tau)$ resulting from the solution of equation (2) should meet the boundary conditions on the surface of the material heated:

$$
-\lambda \frac{\partial T}{\partial n}=\alpha\left(T_{w}-T_{g}\right)
$$

where:

$\alpha-$ effective heat transfer coefficient, $\mathrm{W} /\left(\mathrm{m}^{2} \cdot \mathrm{K}\right)$;

$T_{w}$ - charge surface temperature, $\mathrm{K}$;

$T_{g}$ - furnace atmosphere temperature, $\mathrm{K}$;

The finite element method was applied to solve the heat conduction equation [12]. Bearing in mind the need to obtain high accuracy of the heat conduction model in conjunction with a short computing time, non-linear shape functions were applied [13]. The non-linear shape functions applied in the finite element method were described with Hermite's polynomials [14], which in the model concerned assume the form:

$$
\begin{array}{ll}
G_{1}^{0}\left(\xi_{1}\right)=1-3 \xi_{1}^{2}+2 \xi_{1}^{3} & G_{1}^{1}\left(\xi_{1}\right)=\xi_{1}-2 \xi_{1}^{2}+\xi_{1}^{3} \\
G_{1}^{0}\left(\xi_{2}\right)=1-3 \xi_{2}^{2}+2 \xi_{2}^{3} & G_{1}^{1}\left(\xi_{2}\right)=\xi_{2}-2 \xi_{2}^{2}+\xi_{2}^{3} \\
G_{1}^{0}\left(\xi_{3}\right)=1-3 \xi_{3}^{2}+2 \xi_{3}^{3} & G_{1}^{1}\left(\xi_{3}\right)=\xi_{3}-2 \xi_{3}^{2}+\xi_{3}^{3} \\
G_{2}^{0}\left(\xi_{1}\right)=3 \xi_{1}^{2}-2 \xi_{1}^{3} & G_{2}^{1}\left(\xi_{1}\right)=-\xi_{1}^{2}+\xi_{1}^{3} \\
G_{2}^{0}\left(\xi_{2}\right)=3 \xi_{2}^{2}-2 \xi_{2}^{3} & G_{2}^{1}\left(\xi_{2}\right)=-\xi_{2}^{2}+\xi_{2}^{3} \\
G_{2}^{0}\left(\xi_{3}\right)=3 \xi_{3}^{2}-2 \xi_{3}^{3} & G_{2}^{1}\left(\xi_{3}\right)=-\xi_{3}^{2}+\xi_{3}^{3}
\end{array}
$$

where: $\xi_{1}, \xi_{2}, \xi_{3}-$ local coordinates.

The applied method was described in the paper [13].

\section{Inverse problem}

In the inverse solution the approximating function method was used. In this method the inverse problem solution comes down to finding the error norm minimum, which is the sum of squares of deviations of the measured and calculated temperatures:

$$
E\left(p_{k}\right)=\frac{1}{N T \cdot N P} \sum_{i=1}^{N T} \sum_{j=1}^{N P}\left(\frac{T e_{i}^{j}-T_{i}^{j}\left(p_{k}\right)}{T e_{i}^{j}}\right)^{2}
$$

where:

$p_{k}-$ vector of the unknown parameters,

$N T$ - number of the temperature sensors,

$N P$ - number of the temperature measurements performed by one sensor,

$T e_{i}^{j}-$ the sample temperature measured by the sensor $i$ at the time $\tau_{j}$,

$T_{i}^{j}-$ the sample temperature at the location of the sensor $i$ at the time $\tau_{j}$ calculated from the finite element solution to the heat conduction equation.

The minimum of the error norm was determined with the variable metric method, using the BGFS (Broyden-FletcherGoldfarb-Shanno) algorithm [15].

Approximation of the distribution of average heat flux during heating was improved by dividing the total time into intervals and approximating the heat flux in each interval using parabolic functions in the form:

$$
\begin{gathered}
W_{1}=1-3 \xi+2 \xi^{2} \\
W_{2}=4 \xi-4 \xi^{2} \\
W_{3}=2 \xi^{2}-\xi
\end{gathered}
$$

The sensitivity tests of the inverse model are presented in the paper [13].

\section{Numerical computing}

In order to determine the heat flux distribution, the charge was heated in a laboratory furnace fired with natural gas (Fig. 1). The internal dimensions of the furnace chamber were: $450 \times 550 \times 1340 \mathrm{~mm}$. The furnace had a fibrous lining, and was fitted with 1 kinetic burner with a power of $50 \mathrm{~kW}$. The cubeshaped charge, with a side of $150 \mathrm{~mm}$, was made of $15 \mathrm{HM}$ steel. On each charge wall temperature was measured at 5 points with K-type thermocouples with a thickness of $1 \mathrm{~mm}$, installed at a depth of $3 \mathrm{~mm}$ under the surface. In order to obtain a good representation of actual conditions, the charge was placed on a metal foundation. As a result measurements from the furnace hearth side were also possible. For the purposes of determination of the heat transfer coefficient, the furnace was fitted with additional 6 thermocouples measuring the furnace atmosphere temperature near individual charge surfaces. Indications of 36 thermocouples were recorded with a MGCplus measurement system with an accuracy class of $\pm 0.2 \%$. The maximum error of the measurement system did not exceed $2.5 \mathrm{~K}$.

The furnace was controlled on the basis of readings of the thermocouple located beyond the flame impact zone. Heating was conducted to a maximum furnace temperature of $1165^{\circ} \mathrm{C}$, the 


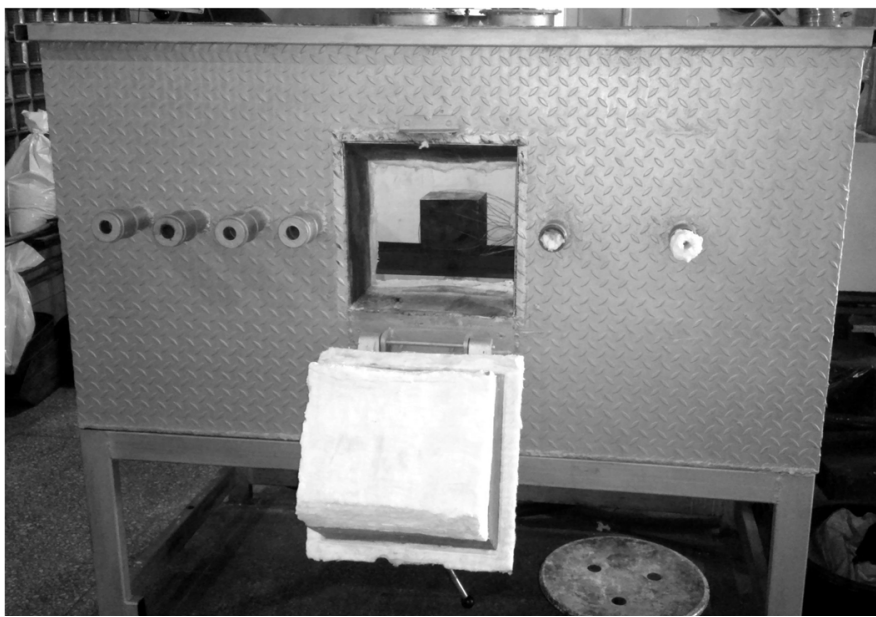

Fig. 1. The test stand

process was aborted after obtaining similar temperature readings by the thermocouples. The heating time was $8800 \mathrm{~s}$.

Inverse method calculations were conducted for a time interval of $5 \mathrm{~s}$ and the division of the charge into 27 elements $(3 \times 3 \times 3)$ with non-linear shape functions. The physical properties of $15 \mathrm{HM}$ steel were set as temperature dependent functions
- Fig. 2. The comparison of the temperature distribution at the bottom and the top surface obtained by measurements with calculation results is presented in Fig. 3. The graphs also include furnace atmosphere temperature distributions near individual charge surfaces. More than satisfactory conformity of the temperature distribution was obtained, the mean square error $\mathrm{dt}$ obtained by calculations was $6.4^{\circ} \mathrm{C}$. The maximum positive and negative temperature deviations were 35.4 and $-21.7^{\circ} \mathrm{C}$. Taking into account temperature fluctuations and its non-uniform distribution within the furnace chamber, the numerical simulation results should be deemed satisfactory

The obtained temperature distributions result from the values of heat flux obtained with inverse calculations, Fig. 4. The maximum values of heat flux at all heated surfaces were observed in the initial period of heating (up to $2000 \mathrm{~s}$ ). For the top surface it is about $80 \mathrm{~kW} / \mathrm{m}^{2}$, at the side surfaces almost $80 \mathrm{~kW} / \mathrm{m}^{2}$, whereas for the bottom surface of $20 \mathrm{~kW} / \mathrm{m}^{2}$. The biggest heat amount in the whole range of the heating process is absorbed at the top surface, after $6000 \mathrm{~s}$ the value of heat flux density drops to about $7 \mathrm{~kW}$, while the other surfaces assume values close to zero after this time. The temperatures of individual surfaces after this heating time reache about $1100^{\circ} \mathrm{C}$.
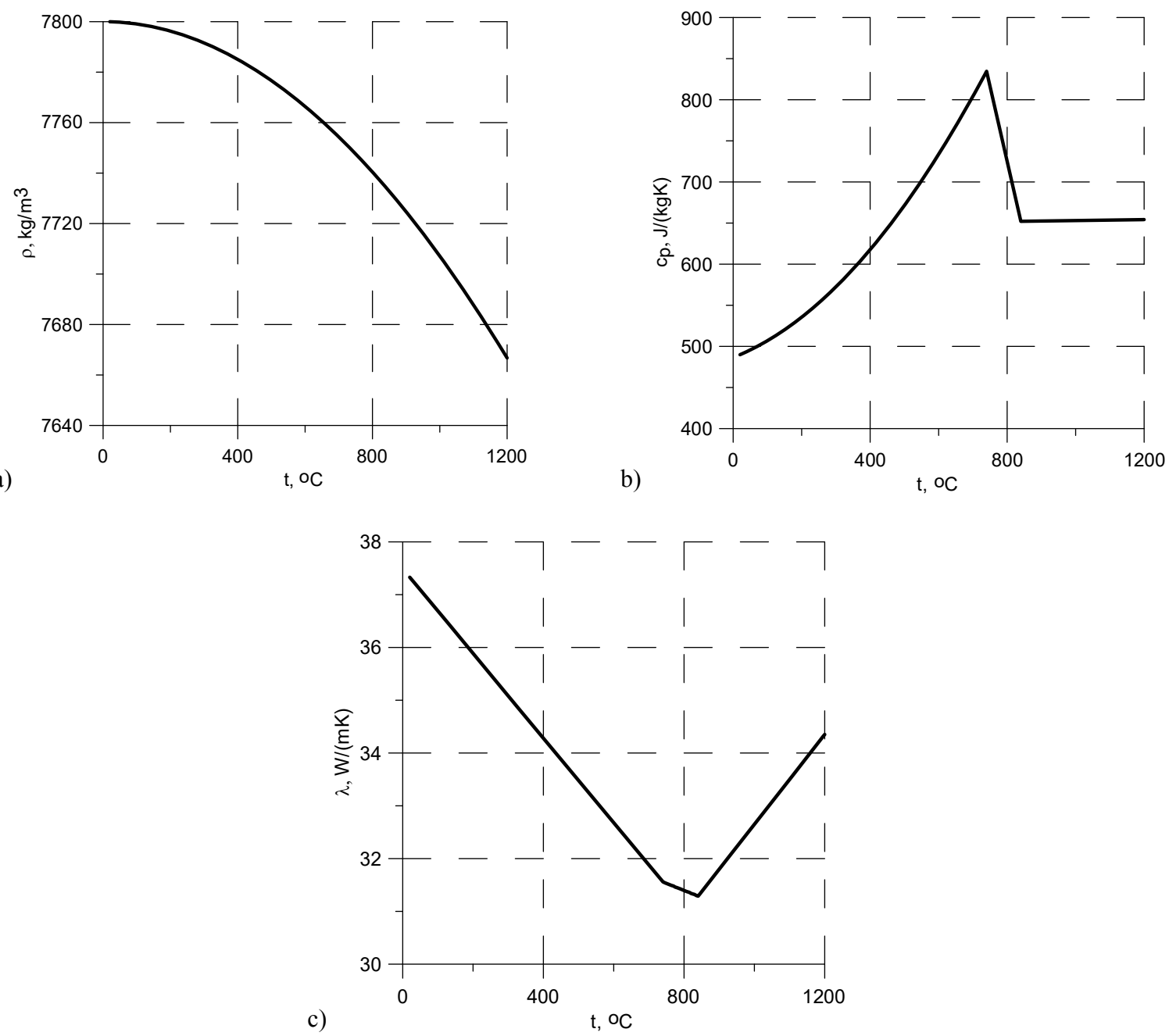

Fig. 2. The physical properties of $15 \mathrm{HM}$ steel: a) density; b) specific heat; c) thermal conductivity 

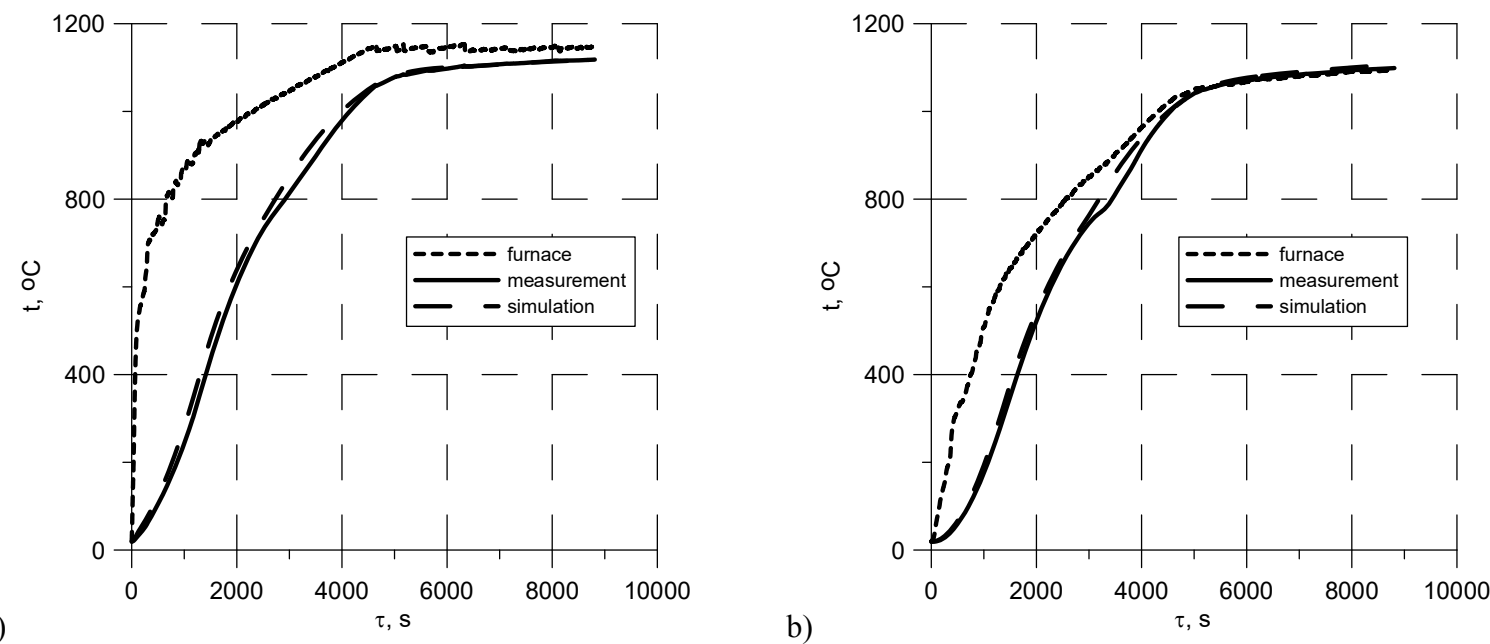

Fig. 3. Comparison of temperature distribution in the characteristic points: a) a top surface; b) the lower surface

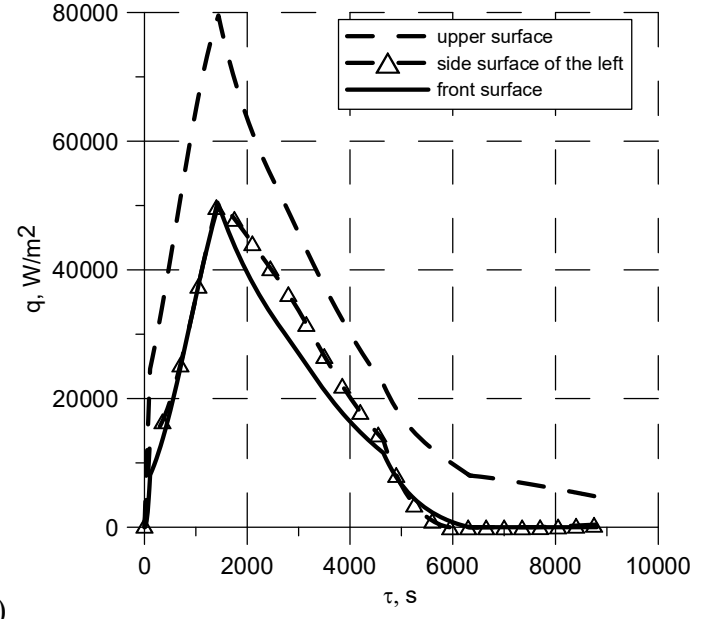

a)

Fig. 4. The heat flux distribution during the charge heating, $15 \mathrm{HM}$ steel

The obtained values of heat flux were used to determine the effective heat transfer coefficient using the Newton law :

$$
\alpha_{e f}=\frac{q_{i n v}}{t_{g}^{k}-t_{w}}
$$

where:

$q_{i n v}-$ heat flux obtained from the inverse analysis, $\mathrm{W} / \mathrm{m}^{2}$;

$t_{g}^{k}$ - furnace atmosphere temperature near the $k$ surface, ${ }^{\circ} \mathrm{C}$;

$t_{w}$ - charge surface temperature, ${ }^{\circ} \mathrm{C}$.

The obtained distributions of the mean value of heat transfer coefficient as a function of the average charge surface temperature for individual surfaces are presented in Fig. 5. The highest value of the heat transfer coefficient, $373 \mathrm{~W} /\left(\mathrm{m}^{2} \mathrm{~K}\right)$, was obtained for the charge surface temperature of $1040^{\circ}$. The lowest values of the heat transfer coefficient were observed at the bottom charge surface, which did not exceed $95 \mathrm{~W} /\left(\mathrm{m}^{2} \mathrm{~K}\right)$. After exceeding the maximum of the heat transfer coefficient, its rapid drop can be clearly observed, however one should bear in mind that within this range the charge surface temperature approaches the furnace atmosphere temperature.

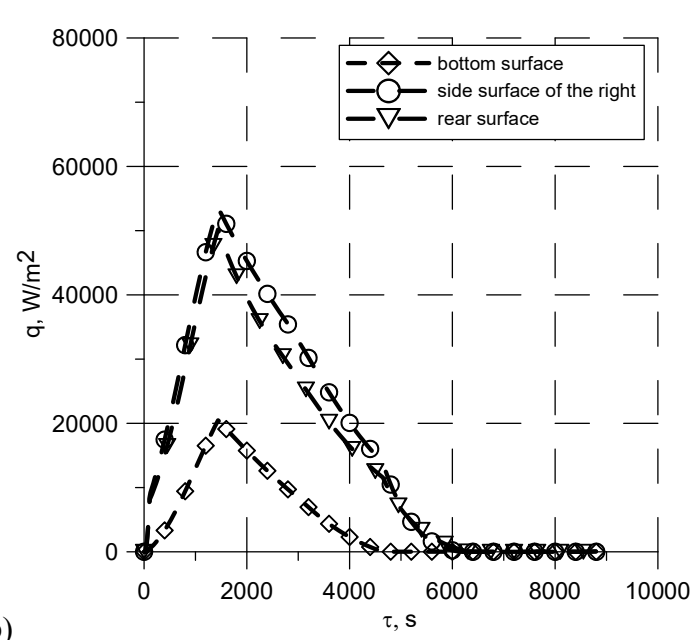

b)

This paper presents a method of identification of heat transfer coefficient at all 6 surfaces of the charge heated. The finite element method was used to solve the heat conduction problem in the charge heated. The inverse method allowed the heat flux to be identified, which then was used to determine the boundary condition. The maximum values of heat flux were observed after about $2000 \mathrm{~s}$ of heating, then the charge started absorbing less and less heat on all surfaces. After this time, the temperature of charge's top surface achieved $600^{\circ} \mathrm{C}$ for the furnace atmosphere temperature of about $980^{\circ} \mathrm{C}$. As it turns out, the effective heat transfer coefficient for this surface temperature is $150 \mathrm{~W} /\left(\mathrm{m}^{2} \mathrm{~K}\right)$. The effective heat transfer coefficient reaches its maximum values only at a temperature of about $1040^{\circ} \mathrm{C}$, or just before the time when the heat flux starts reaching nearly zero values. It is likely to be related to heating the charge and the furnace walls to adequately high temperatures, which prevents further charge heating. 

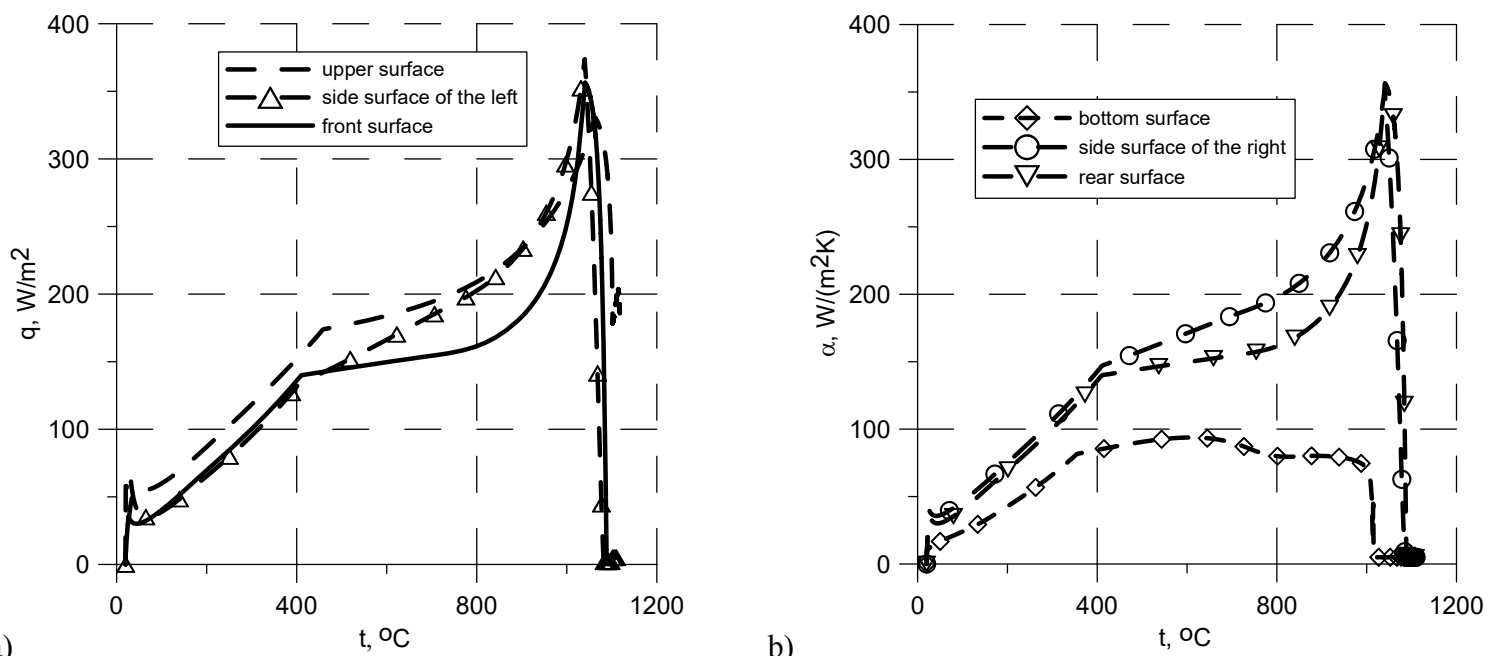

Fig. 5. The heat transfer coefficient distribution during the charge heating, $15 \mathrm{HM}$ steel

\section{Acknowledgements}

Final remark: Scientific study financed from the regular activity of the Faculty of Metals Engineering and Industrial Computer Science of AGH University of Science and Technology.

\section{REFERENCES}

[1] H.Y. Li i M.N. Ozisik, Journal of Heat Transfer, Transactions of the ASME 114, 1060-1063 (1992).

[2] C.E. Siewert, Journal of Quantitative Spectroscopy and Radiative Transfer 52 (2), 157-160 (1994).

[3] K. Kazuhiko i in., Bulletin of the JSME Series B. 39 (4), 808-814 (1996).

[4] H.M. Park i D.H. Yoo, International Journal of Heat and Mass Transfer 44, 2949-2956 (2001).

[5] O.M. Alifanov, Inverse heat transfer problems, 1994 Springer-Verlag.

[6] R. Fletcher, Practical methods of optimization, 1987 John Wiley \& Sons.
[7] H.J. Reinhardt, International Journal for Numerical Methods in Engineering 32, 363-383 (1991).

[8] N. Hamidreza, K.A. Woodbury, J. Beck, International Journal of Heat and Mass Transfer 91, 1148-1156 (2015).

[9] A. Sugianto i in., Journal of Materials Processing Technology 209, 4627-4632 (2009).

[10] H.K. Kim , S.I. Oh, Journal of Materials Processing Technology 112, 157-165 (2001).

[11] H.M. Park, W.J. Lee, Chemical Engineering Science 57, 20072014 (2007).

[12] Z. Malinowski, Numeryczne modele w przeróbce plastycznej i wymianie ciepła, 2005 Uczelniane Wydawnictwa Naukowo-Dydaktyczne.

[13] Z. Malinowski i in., International Journal of Heat and Mass Transfer 75, 347-361 (2014)

[14] O.C. Zienkiewicz, R.L. Taylor, J.Z. Zhu, The Finite Element Method: Its Basis and Fundamentals, 2013 Butterworth-Heinemann.

[15] W. Findeisen, J. Szymanowski, A. Wierzbicki: Teoria i metody obliczeniowe optymalizacji, 1980 PWN. 